\section{OP03 COMPARATIVE ANALYSIS OF RISK FACTORS FOR EXCESS AND USUAL MORTALITY DURING THE FIRST 2020 COVID-19 PANDEMIC PHASE IN ENGLAND*}

Iain Carey*, David Strachan, Derek Cook, Tess Harris, Stephen DeWilde, Umar Chaudhry. Population Health Research Institute, St George's, University of London, London, UK

\subsection{6/jech-2021-SSMabstracts.3}

Background The first wave of the COVID-19 pandemic in England resulted in an approximate 50\% increase in all-cause mortality during mid-March to mid-May 2020. Previous analyses have studied COVID-related deaths, but these were underrecorded early in the pandemic due to paucity of virological testing. We propose a novel method to identify excess mortality during the pandemic by accounting for usual all-cause mortality in pre-pandemic years using a large electronic primary care database. We estimated the impact of risk factors (RFs) on excess mortality during the first wave and compared these with the impact of the same RFs on total mortality in nonpandemic times.

Methods An average of 4.8 million patients aged 30-104 years active in 770 CPRD Aurum practices between 18th March and 19th May over a 6-year period (2015-2020) were included. Concurrent medical history, ethnicity, area-based deprivation and fatalities were extracted for each year. Poisson regression models fitted total mortality adjusting for age and sex, with interactions between each RF and the pandemic and reference periods. Total mortality during the pandemic was partitioned into 'usual' and 'excess' components, assuming 2015-19 rates represented 'usual' mortality. The association of each RF with the 2020 'excess' component was derived as the excess mortality ratio (EMR), to compare with the usual mortality ratio (UMR).

Results RFs where excess mortality was greatest and notably higher than usual were age $>80$, black ethnicity, BMI $>40$, dementia, learning disability, London practices and people in care-homes. For example, people of black ethnicity vs. white had an $\mathrm{EMR}=2.50$ (95\%CI 1.97-3.18) compared to a $\mathrm{UMR}=0.92 \quad(95 \% \mathrm{CI} 0.85-1.00)$. Excess mortality was more comparable to usual mortality for sex and area deprivation. The EMR for men $(1.46$, 95\%CI 1.32-1.60) was not significantly different than the $\mathrm{UMR}=1.34 \quad(95 \% \mathrm{CI}$ 1.32-1.37). The EMR $=2.05$ (95\%CI 1.76-2.38) in the most deprived quintile (vs. least) was only slightly higher than the $\mathrm{UMR}=1.70 \quad(95 \% \mathrm{CI} 1.65-1.75)$. Although some RFs produced EMRs significantly lower than their UMRs (Cancer, COPD), the EMRs were still $>1$. However, current smoking was inversely associated with excess mortality: while current smokers were $64 \%$ more likely to die than non-smokers in 2020, when the UMR $=2.12$ (95\%CI 2.072.18) was accounted for, the $\mathrm{EMR}=0.80$ (95\%CI $0.65-$ $0.98)$.

Conclusion Utilising large electronic patient databases to study trends in excess mortality during the pandemic confirmed some reported findings (e.g. ethnicity, obesity and care-homes), but also highlighted important differences not apparent from studying cause specific mortality during the pandemic (e.g. smoking, sex and area deprivation).

\section{OP04 \\ OWNERSHIP AND COVID-19 IN CARE HOMES FOR OLDER PEOPLE: A LIVING SYSTEMATIC REVIEW OF OUTBREAKS, INFECTIONS, AND MORTALITY*}

${ }^{1}$ Anders Bach-Mortensen*, 'Michelle Degli Esposti, 'Ben Verboom, ${ }^{2}$ Ani Movsisyan. 'Social Policy and Intervention, University of Oxford, Oxford, UK; ${ }^{2}$ Pettenkofer School of Public Health, LMU Munich, Munich, Germany

\subsection{6/jech-2021-SSMabstracts.4}

Background The adult social care sector is increasingly outsourced to for-profit providers, who constitute the largest provider of care homes in many developed countries. During the COVID-19 pandemic, for-profit providers have been accused of failing their residents by prioritising profits over care, prevention, and caution, which has been reported to result in a higher prevalence of SARS-CoV-2 infections and deaths in forprofit care homes. However, the growing body of academic research investigating ownership variation across COVID-19 outcomes has not been systematically synthesised. We aimed to identify, appraise, and synthesise the available research on ownership variation in COVID-19 resident and staff outcomes (outbreaks, infections, deaths, shortages of personal protective equipment (PPE) and staff) across care homes for older people, and to update our findings as new research becomes available.

Methods This living systematic review was prospectively registered with PROSPERO (CRD42020218673) and on OSF (https://osf.io/c8dq9/). We searched 17 databases and performed forward and backward citation tracking of all included studies. Search results were screened and reviewed in duplicate. Risk of bias (RoB) was assessed in duplicate according to the COSMOS-E guidance. Data were extracted by a single review author and independently validated by a second. The results were synthesised by country, RoB, and model adjustments, and visualised using harvest plots.

Results Twenty-nine studies across five countries were included in the first iteration of this review, with $75 \%$ of included studies conducted in the Unites States. For-profit ownership was not consistently associated with a higher probability of a COVID-19 outbreak. However, there was compelling evidence of worse COVID-19 outcomes following an outbreak, with for-profit care homes having higher rates of accumulative infections and deaths. For-profit care homes were also associated with a number of risk factors, such as crowdedness, size, client vulnerability, inferior quality ratings, and PPE shortages, which may have contributed to the higher incidence of infections and deaths.

Discussion Understanding and analysing systematic variation across ownership groups is of immense policy relevance, given that the vast majority of care homes in many developed countries are for-profit entities. Our synthesis demonstrates that for-profit ownership and associated characteristics were consistent risk factors for higher cumulative COVID-19 infections and deaths in the first wave of the pandemic. Thus, ownership and the characteristics associated with for-profit care home providers may present key regulatable factors that can be addressed to improve health outcomes in vulnerable populations and reduce health disparities. 